\title{
Transmission of Medical Images over Multi-Core Optical Fiber using CDMA: Effect of Spatial Signature Patterns
}

\author{
Antoine Abche ${ }^{1} \quad$ Boutros Kass Hanna ${ }^{2} \quad$ Lena Younes $^{2}$ Nour Hijazi $^{2} E l i e$ Inaty ${ }^{1}$ Elie Karam ${ }^{1}$ \\ ${ }^{1}$ Electerical Engineering Department, University Of Balamand, Lebanon, \\ \{antoine.abche, elie.inaty, elie.karam\}@balamand.edu.lb \\ ${ }^{2}$ Computer Engineering Department, University Of Balamand, Lebanon, \\ \{boutros.kasshanna, lena.younes, nour.hijazi\}@std.balamand.edu. Ib
}

\begin{abstract}
In this work, the effect of the 2-D Optical Orthogonal Spatial Pattern Codes (OOSPC) is evaluated quantitatively for the transmission of medical images over Multi-core optical fiber using a double blind CDMA technology. The implemented method assumes that $\mathrm{P}$ medical practitioners or users are working simultaneously and transmitting images from one site to another. The transmitted images are encoded using a two-steps procedure: 1) coding the pixels (users) using a particular OOSPC and 2) coding the bits using time orthogonal basis functions. The encoding procedure follows the decomposition of an image into its bits to increase the transmission rate by performing a parallel transmission. Then, the encoded information from different images is combined using a multiplexer and is transmitted over the multi-core optical fiber. The transmitted information is de-multiplexed at the receiver side to identify the user that is transmitting the information and consequently, to reconstruct the original image i.e. is decoded using the same double blind Orthogonal Signatures. The performance is quantitatively evaluated using Monte-Carlo simulation techniques using different criteria, namely, the Performance Test, The Bit Error Rate, the Root Mean Square Error and the Pixel Error Rate.
\end{abstract}

Keywords: medical image transmission, CDMA, bit error rate, orthogonal spatial signature, fiber optics

\section{Introduction}

Over the past years, the transmission of image data over various transmission or link mediums (such as cables, local area networks, wide area networks, wireless communications and Fiber optics) has gained a big momentum [Kohli, 1989;Tsiknakis et.al., 1996]. The transmission of data, especially of a large number of images, within the same medical facility or between facilities from one site to another is of great importance in order to visualize, process and/or analyze the medical information and consequently to achieve a better diagnosis by various medical practitioners. In this context, the exchange of information will lead to the improvement of the health and the quality of the patient's life. Besides, the cost of the health care can be reduced, especially for patients who live in rural regions.

These reasons have given the impetus and the driving force to research groups and data service providers to concentrate their works in this area. Furthermore, the research has been geared toward the transmission of data using multiple access transmission technologies such as Time Division Multiple Access (TDMA) and Frequency Division Multiple Access (FDMA) [Kohli, 1989; Tsiknakiset.al. 1996]. However, the latter techniques require very sophisticated network management and scheduling approaches [Kohli, 1989; Tsiknakiset.al., 1996]. Thus, Code Division Multiple Access (CDMA) approach has been used in image transmission to mitigate and/or to reduce such complexities [Tsiknakis et.al., 1996; Chang et. al., 1998; Abtahi et.al., 2002; Kitayana, 1994; Lisimachos et. al., 2005; Chang et. al. , 1996; Kamakura et. al., 2003; Peng et. al. , 2008; Yang et.al., 2011]. The techniques introduced in references [Tsiknakis et.al.,1996; Chang et. al., 1998; Abtahi et.al., 2002; Kitayana, 1994; Lisimachos et. al., 2005; Chang et. al. , 1996; Kamakura et. al., 2003] have used a spatial CDMA approach which in turn ensures a fast transmission. However, they have failed to preserve the pixel's intensities of the transmitted image at the receiver. The quality of the reconstructed image is of great importance in healthcare and medical applications in which a large number of images can be transmitted between various sites. That is because any distortion or error in the associated received images would lead to an inaccurate diagnosis and analysis. Consequently, wrong decisions will be made by various medical practitioners and could greatly affect the patient's life.

The CDMA-based approach has several advantages. The same range of time or the same frequency bandwidth can be occupied by several users simultaneously. Therefore, high quality images can be reconstructed using the highest spectrum efficiency in conjunction with several spatial Optical Orthogonal 
Signature Patterns (OOSPs) [Yang et. al., 1998]. Consequently, the large bandwidth of the optical fiber will be an advantage in image transmission which will improve the quality of the patient's diagnostic. However, the information (data, images) should be transferred authentically and with a sufficient accuracy. Therefore, a multi access approach characterized by high speed, high spectrum efficiency and high quality data transmission rate is required for medical images' transmission. Thus, a double blind CDMA technique using a multi-core optical fiber was introduced to improve the reconstructed image's quality at the receiver end and to increase the transmission of the data rate [Abche et.al., 2011] between the various medical facilities. In this work, the effect of 2D spatial OOSPC on the latter CDMA based approach is studied.

This paper is organized as follows: the system model is presented in Section II. The method of evaluation using Monte Carlo simulation techniques is given in Section III. Also, different criteria to study its performance are introduced. The corresponding results are presented, analyzed and discussed in Section IV and a conclusion is given in Section V.

\section{System Model}

Figure 1 illustrates the implemented OCDMA approach to investigate the effect of $2 \mathrm{D}$ spatial OOSPCs on the transmission of images. It is assumed that $\mathrm{P}$ healthcare practitioners (users) are transmitting simultaneously medical images from one location to another over a multi-core optical fiber. Each image consists of $\mathrm{M} \times \mathrm{N}$ pixels and the pixel's intensity is represented by $\mathrm{n}$ bits. The optical encoding (temporal and spatial) and decoding of the images constitute the principal concept of the CDMA based technique [Abche et.al., 2011].

The encoding operation requires the decomposition of each image into n-binary images using the Bit Plane Decomposition (BDP) technique [Gonzalez et.al., 2008]. That is, one image is generated from the least significant bit (Bit 0) of each pixel's intensity. Another binary image consists of bit 1 of each pixel's intensity. Similar images are generated for bit 2 until bit $n$ (i.e. the most significant bit).

The next step involves the temporal encoding of each pixel's intensity. That is, the bits are converted from serial-to-parallel for transmission purposes. Each bit is converted to an optical signal using a broadband light source (such as a light emitting diode). Then, each generated ultra-short light pulse is temporally encoded using a sequence of optical delay lines and is selected according to pre-determined basis functions. The results of each pixel's temporal encoding process (from bit 0 to bit $\mathrm{n}$ ) are multiplexed using a passive optical coupler.

Then, the output of the multiplexer is spatially encoded using a particular 2-D spatial signature
(OOSP). This procedure is crucial for user's identification by using a specific optical mask. The orthogonality between spatial signatures (OOSPs) will impose certain conditions on the spatial correlation (auto or/and Cross) in order to distinguish one 2-D spatial signature from the other signatures [G-C Yang et.al., 1998]. This double blind approach ensures the ultra high transmission rate and the accuracy needed in most medical imaging applications. The results of the spatial encoding procedure from all users are combined using a multiplexer and the corresponding information is transmitted over the multi-core fiber.

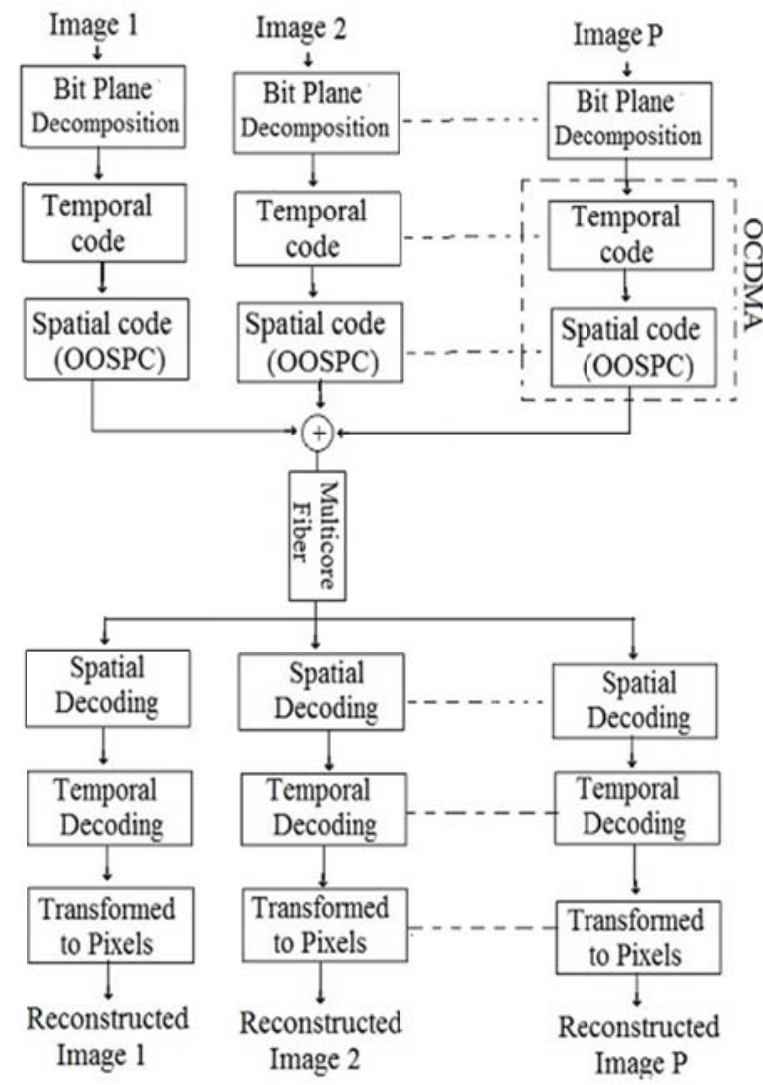

Figure 1.The implemented OCDMA approach.

At the receiver end, the signal is passively demultiplexed using a passive optical splitter. It is fed to a set of optical decoders to identify the users, to extract the corresponding information and to reconstruct the original images. The decoding process involves the spatial decoding of the information using the same spatial mask that is used for encoding purposes. The identification of the desired user is based on the autocorrelation with the desired pixel and the crosscorrelation with the interfering pixels. Then, the corresponding information is fed as inputs to $n$ branches in which the desired signal is decoded by the same encoding temporal code. Each branch corresponds to a particular bit of the desired n-bit pixel. The decoders' outputs are converted to electrical signals using photo-detectors. Finally, the estimated 
bits are converted from parallel-to-serial to form the pixels' intensities.

\section{Method of Evaluation}

The performance of the implemented OCDMA approach is quantitatively evaluated using Monte-Carlo simulation techniques. The simulated images (desired and the interfering) are generated randomly. The intensity of each pixel is represented by 8-bits and each bit is generated uniformly and randomly. That is followed by decomposing each image using the BPD approach into 8 binary images and a temporal coding is generated according to the Galois Field (GF(11)). Then, the image is encoded spatially using a 2-D OOSPC which is selected randomly from a pool of generated signatures. The OOSPCs are generated by assuming the size of the core fiber to be $p$ by $p$ (in this work $p=7$ ) [Yang et. al., 1998]. The pool consists of OOSPCs with a Cross Correlation (CC) and an AutoCorrelation (AC) of zero, one and/or two. Each 2-D signature is assigned to a specific image i.e. user. Having combined the information from the desired and interfering images, the results are transmitted over the optical fibers. At the receiver, the information is collected and the user is identified by performing the correlation of the desired and received patterns. Then, the coded bit stream is extracted by correlating the received data with the temporal codes that are implemented at the transmitter. Subsequently, the transmitted image is reconstructed and is evaluated quantitatively by comparing the latter with the original image because it is hampered by users' interference.

In this work, various 2-D spatial patterns are implemented to compare their effects on the transmission of medical images: the Yang \&Kwong approach [Yang et. al., 1998], the Extended Hyperbolic Congruential Hop Code (EHC) [Wronskiet.al., 1996] and several versions of The Frequency-Hopped Spread Spectrum (FHSS) code [Shaar et. al., 1984.]. The performance of the presented approach is quantitatively evaluated using several similarity measures: the Bit Error Rate (BER), the pixel Error Rate (PER), the Root Mean Square Error (RMSE) and the Performance Test (PT).

\subsection{Bit Error Rate (BER)}

In image transmission, the BER can be defined as the percentage of bits that are transmitted in error with respect to the total number of bits. For example, a transmission with a BER of $10^{-6}$ means that one bit is in error when 1,000,000 bits are transmitted.

\subsection{Pixel Error Rate (PER)}

As it is mentioned earlier, an image consists of MxN pixels and each pixel consists of several bits. Therefore, if one bit is transmitted in error, the corresponding intensity value of the pixel will differ from its original value. In medical applications, the quality (integrity) of the received image is of great value and importance because any variation of the pixels' intensities can lead to a different appearance and consequently medical practitioners can provide an inaccurate diagnosis. Therefore, if the pixel's intensity is transmitted with an error; the reconstructed values of the received image will vary from the corresponding original intensity values. Besides, the error's severity depends on the bit's location that is transmitted in error over the multi-core optical fiber (bit 0-the least significant bit, bit $1 \ldots$ bit $\mathrm{n}$-the most significant bit).

\subsection{Root Mean Square Error (RMSE)}

The RMSE is a quantitative criterion to measure the performance of a particular approach [Gonzalez et. al., 2008]. The measure is defined in terms of the difference between the original image $(I(i, j))$ and the reconstructed image $\left(\mathrm{I}_{\text {recons }}(\mathrm{i}, \mathrm{j})\right)$ at the receiver end:

$$
R M S E=\sqrt{\frac{1}{M N} \sum_{i=1}^{N} \sum_{j=1}^{M}\left(I(i, j)-I_{\text {recons }}(i, j)\right)^{2}}
$$

\subsection{Performance Test (PT)}

The Performance Test (PT) is a quantitative measure to evaluate the similarity of two images. It is defined in terms of two reference images: "a high quality image ( $\left.\mathrm{I}_{\text {full }}\right)$ and a low quality image ( $\left.\mathrm{I}_{\text {worst }}\right)$ ". The high quality image is assumed to be the original image and the latter is the objective of the transmission approach. On the other hand, the worst image is defined as to be an image where all transmitted bits are wrong. The PT is defined as [Fuderer, 1989]:

$$
P T=1-\frac{\left\|I_{\text {full }}-I_{\text {recons }}\right\|^{2}}{\left\|I_{\text {full }}-I_{\text {worst }}\right\|^{2}}
$$

If the reconstructed image is similar to the original image, it is evident that the PT measure yields a value of 1 . If the reconstructed is similar to the worst image, the PT has a value of 0 . Therefore, the closer the reconstructed image is to the original image, the closer the PT value is to 1 and the better is the performance of the tested approach. Thus, the PT measure varies between 0 and 1 depending on the accuracy of the reconstructed image.

\section{Results and Discussion}

In this section, the results of the performed simulations are presented. The study is performed quantitatively using Monte-Carlo simulation Techniques. First, the principal 2-D Optical Orthogonal Spatial Pattern Codes (OOSPC) are generated by implementing the appropriate equations for each code-generation approach. Then, a pool (or pools) of 2-D spatial patterns can be constructed from the initial principal set by performing a row and/or a column shift. At this 
stage, the pool consists of all the generated signatures that are correlated i.e. a pool could contain all the signatures that are characterized by an auto-correlation $\lambda \mathrm{a}$ of 0 and/or 1 and a cross-correlation $\lambda \mathrm{c}$ of 0 and/or 1. Consequently, the latter patterns (i.e. OOSPCs) are kept and each one will be assigned to identify a particular user (i.e. doctor) who is transmitting medical information (such as three dimensional images) over the multi-core optical fiber using CDMA approach. Thus, various pools can be generated based on the values of the auto-correlation and/or the cross correlation.

In this context, Figure 2 shows the four principal OOSPC's that are constructed using Yang and Kwong's approach and from which various pools of 2D patterns can be generated [Yang et. al., 1998]. It is assumed that the medical images are transmitted over a 7 by 7 Multi-core Optical fiber. In each pattern, a white pixel reflects that the information is transmitted through the corresponding optical fiber. These four patterns are characterized by a correlation of zero. The generation requires the definition of two parameters and a prime number $\alpha$ in order to execute the corresponding equations [Yang et. al., 1998]. Similarly, the 2-D signatures of the other techniques are generated and are implemented in this work for comparison purposes.

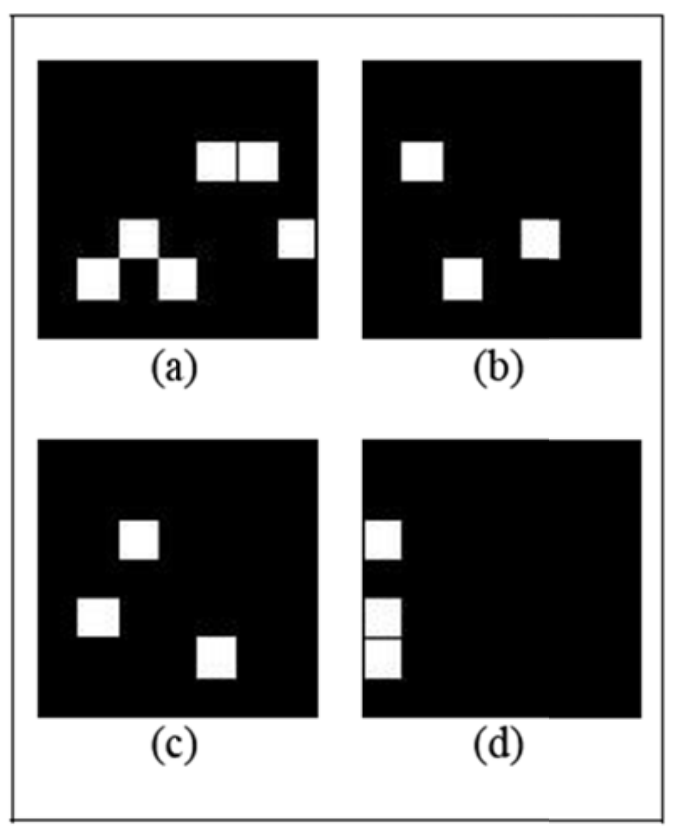

Figure 2.The principal codes generated using YangKwong's approach.

Figures 3, 4, 5 and 6 show the dependence of the BER, the PER, the RMSE and the PT on the number of users $\left(\mathrm{N}_{\mathrm{i}}\right)$ who are transmitting information simultaneously, respectively. Each figure shows the display of five plots. Each plot corresponds to a different approach: Yang-kwong (black color) EHC (blue color), FHSS-1 (red color), FHSS-2 (green color) and FHSS-3 (yellow color). The 2-D spatial signatures are divided into two pools. The first pool (Pool A) includes all the signatures that have a correlation of 0 or 1 . The remaining patterns (correlation $=2$ ) are included in the second pool (Pool B). As is already stated, the signatures are selected randomly from the first pool and then they are selected randomly from the second pool when the first pool is empty.

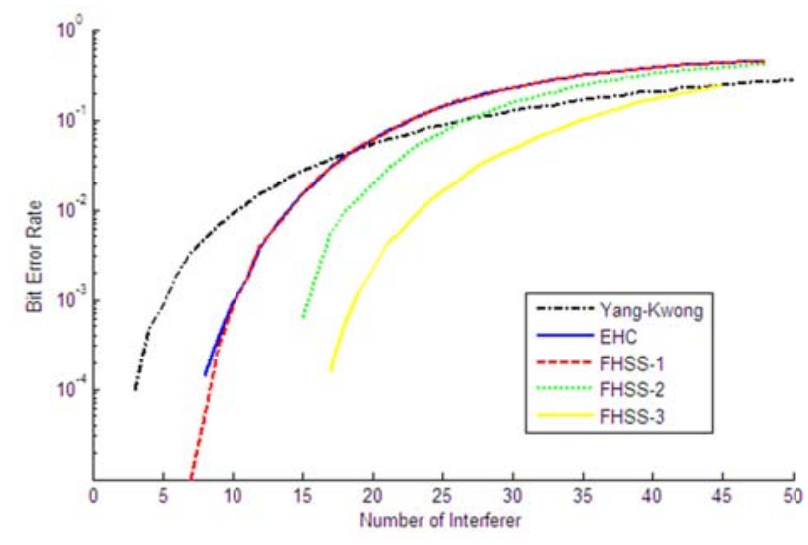

Figure 3.Dependence of BER on $\mathrm{N}_{\mathrm{i}}$ for Various 2-D OOSPCs.

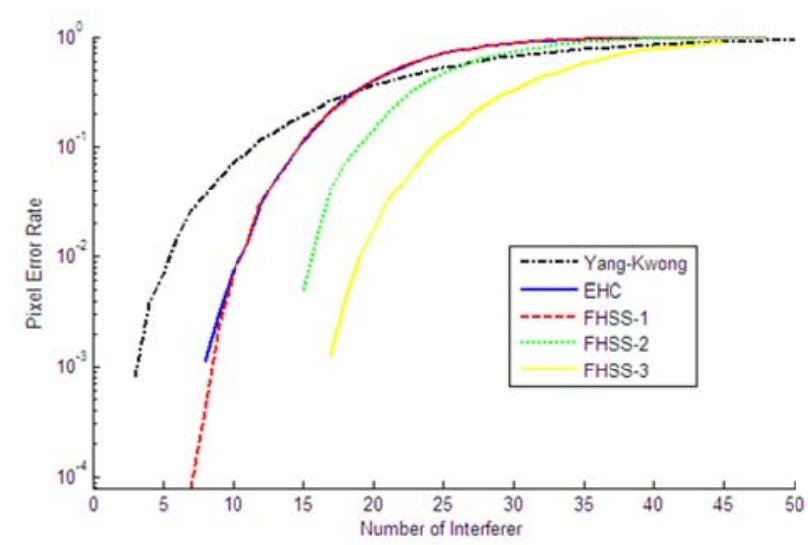

Figure 4.Dependence of the PER on $\mathrm{N}_{\mathrm{i}}$ for Various 2-D OOSPCs.

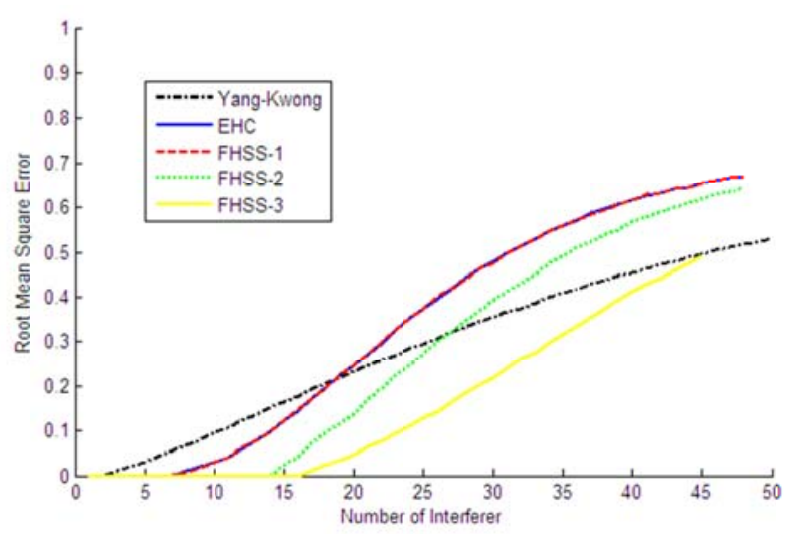

Figure 5.Dependence of the RMSE on $\mathrm{N}_{\mathrm{i}}$ for Various 2-D OOSPCs.

The results of the Monte Carlo simulations illustrate the following: 
i) The RMSE, BER and PER increase as the Number of users $\left(\mathrm{N}_{\mathrm{i}}\right)$ transmitting over the multi-core fiber is increased. This is reflected in a higher error value. Similarly, as $\mathrm{N}_{\mathrm{i}}$ is increased, the PT is decreased i.e. the received image is closer to the worst image. Thus, intensities of the desired image are becoming more and more different from the original intensities. This observation is associated with each 2D OOSPC code.

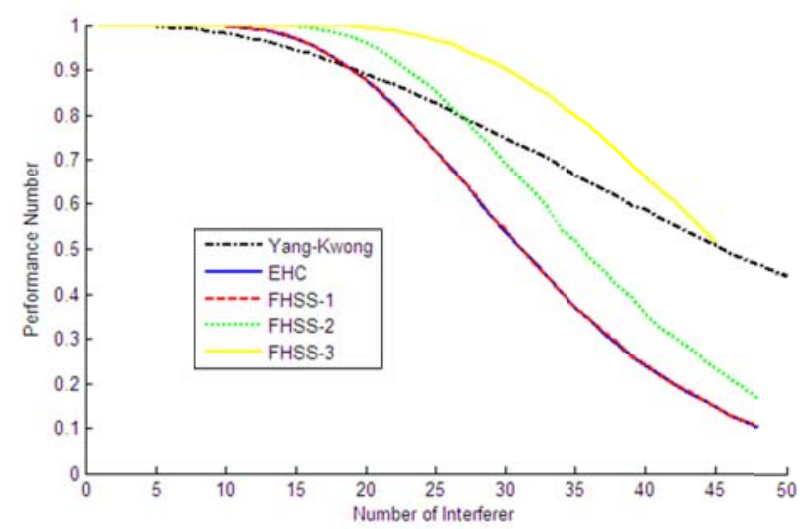

Figure 6.Dependence of the Performance Number on $\mathrm{N}_{\mathrm{i}}$ for Various 2-D OOSPCs.

ii) The EHC and FHSS-1 based OCDMA approaches exhibit similar results for BER, PER, PT and RMSE. Besides, for a given number of users, the BER, PER, RMSE and the PT have different errors for various 2-D OOSPC codes.

iii) A transmission error is observed when 3 users are sending images simultaneously in conjunction with the desired user using the Yang-Kwong approach. On the hand, the FHSS-2 algorithm and FHSS-3 algorithm preserve the integrity of the transmitted information with 14 (FHSS - 2) and 16 interferers (FHSS - 3), respectively (i.e. $\mathrm{BER}=0, \mathrm{PER}=0, \mathrm{RMSE}=0$ and $\mathrm{PT}$ $=1$ ). Thus, it is evident that the FHSS-3 algorithm shows the best results among the five algorithms and consequently, the best encoding scheme (in this work) to send medical images over the transmission medium and to preserve the quality of these images. Also, the FHSS-2 algorithm is the second best code until 25 users are transmitting simultaneously. However, it has to be kept in mind that the FHSS-3 algorithm generates 2-D 8x8 Spatial Patterns whereas the FHSS2 algorithm generates 2-D 7x7 signatures. This might explain the higher number of users (16 instead of 14). For completion purposes, the transmission without an error can be achieved with 7 and 8 interferers using the EHC and the FHSS-1 spatial signatures, respectively.

iv) While EHC and the FHSS-1 algorithms are the worst approaches when the number of interferers is greater than 17, the Yang-Kwong approach exhibits the worst error for Ni less than 17. That is reflected in a higher BER (Figure 3), a higher PER (Figure 4), a higher RMSE (Figure 5) and a lower performance number (Figure 6). This could be due to the fact that the corresponding 2-D OOSPCs have a higher crosscorrelation.

v) The transmission error can be associated with the cross correlation at the receiver end in order to identify the desired user and the selection of the weight or threshold value. Therefore, it might be that the cross correlation differs from one family of codes to another.

As is already stated, the 2-D OOSPCs are selected from two pools and the correlation is the factor that determines the signatures in each pool. In this Monte Carlo experiment, a third pool (Pool C) is formed and it consists of all the signatures (Pool A and Pool B). Consequently, the signature is selected randomly from the pool $\mathrm{C}$ and is assigneid to the user as outlined earlier (referred to as Scenario 1). Consequently, the corresponding results of each technique are compared with the results under the condition that the signatures are generated from Pool A and then from Pool B (referred to as Scenario 2). Figures 7 and 8 show the Bit Error Rate and the RMSE as a function of the number of interferers, respectively. The Double Blind CDMA approach is implemented with the incorporation of the FSSS-2 spatial encoding. Each figure illustrates two plots. While the first plot corresponds to Scenaiol (blue color), the second reflects the results of Scenario 2 (green color).

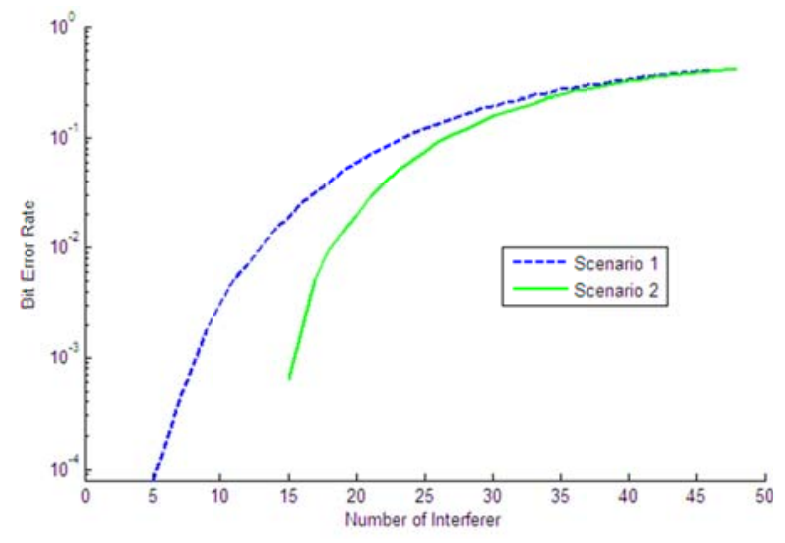

Figure 7. Dependence of BER on the selection of signatures for various number of interferer (FHSS-2 algorithm).

The results clearly show that the transmission of medical images is achieved more accurately using Scenario 2. That is, the integrity of the transmitted data is more preserved. First, as long as the number of users is less than six, the information is transmitted without an error under Scenario 1. On the other hand, fourteen users can transmit data simultaneously without any error at the receiver end. In other words, the reconstructed images will be exactly similar to the original transmitted images. This is due to the fact that the selected signatures have smaller correlation values and consequently the identification of the user is much easier. Second, for a given number of users, the BER and the RMSE values are higher for Scenario 1 than for Scenario 2. This observation remains until more than 


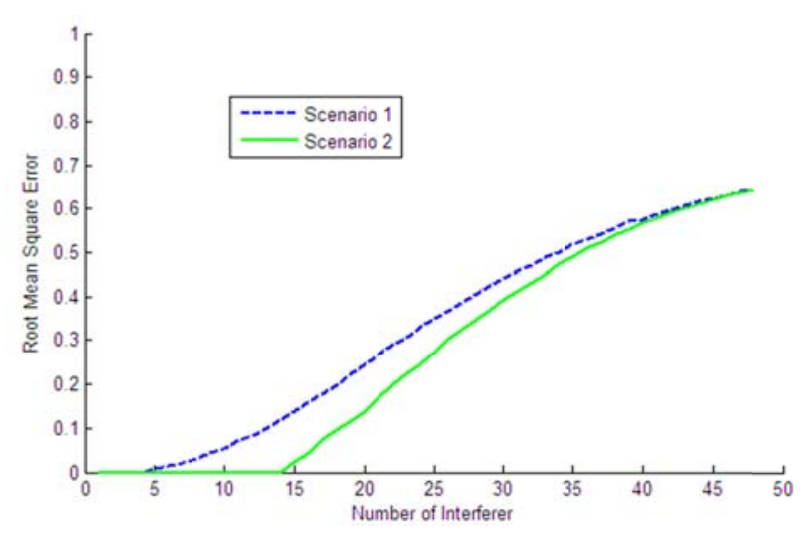

Figure 8.Dependence of RMSE on the selection of signatures for various number of interferer (FHSS-2 algorithm).

forty users are transmitting information at the same time i.e. the values of BER and RMSE become almost the same. Similar observations can be deduced for the dependence of PER and the Performance Test on the number of interferers using the FHSS-2 encoding scheme.

In the same context, Figures 9 and 10 illustrate the effect of the two scenarios on the BER and the RMSE for various numbers of users using the 2-D spatial pattern encoding FHSS-3, respectively. The corresponding results lead to the same conclusions that have been stated when the simulated images are transmitted over multi-core optical fiber and encoded using the FHSS-2 2-D spatial codes. In other words, Scenario 2 provides better results than Scenario 1. That is, the number of users working without any transmission errors is higher for the second scenario (15 vs 5) and the errors associated with scenario 2 are lower for a given number of users. Unlike the FHSS-2 based transmission approach, it can be observed that different errors exist between the two scenarios for a higher number of users. However, as it is stated earlier, this might be due to the fact that the simulated images are assumed to be transmitted over a 7 by 7 multi-core optical fiber using FHSS-2 code and over an 8 by 8 using the FHSS-3 code.

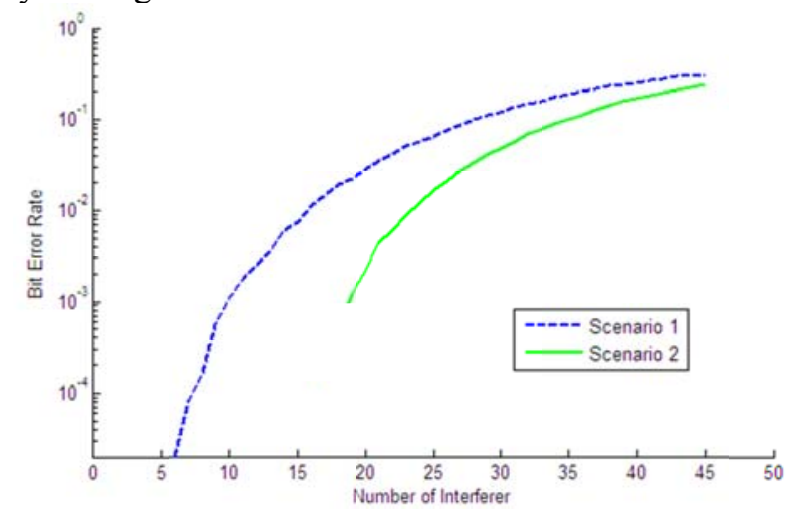

Figure 9.Dependence of BER on the selection of signatures for various number of interferer (FHSS-3 algorithm).

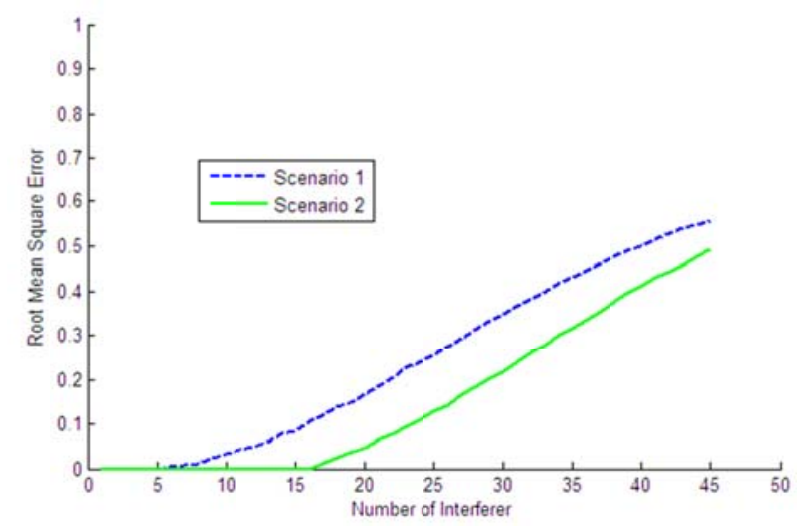

Figure 10.Dependence of RMSE on the selection of signatures for various number of interferer (FHSS-3 algorithm).

\section{Conclusions}

In this work, the effect of the 2-D OOSPCs on the transmission of medical images using a double blind CDMA based technology is investigated. The approach provides fast transmission rates through spatial coding and preserves the simplicity and pixels' information through the temporal coding of the pixels' intensities. It is assumed that $\mathrm{P}$ users are working and transmitting information (i.e. medical images) simultaneously from one site to another within the same facility or between different facilities. The main concepts of the double blind CDMA approach are the optical encoding (temporal and spatial) and the optical decoding of the images. The performance is quantitatively evaluated using Monte Carlo simulation techniques. Several measures (Bit Error Rate, Pixel Error Rate, Root Mean Square Error and Performance Test) are computed for comparison purposes. The OCDMA transmission technique yields better results when the 2-D spatial FHSS-3 or FHSS-2 signatures are incorporated and are assigned to the medical practitioners who are transmitting the corresponding information. The latter conclusion is reflected in lower values of BER, PER and RMSE and a higher value of PT for a given number of interferers as well as in the number of users for which the error of transmission is zero. Besides, it is highly recommended to select first the signatures from a pool that are characterized by a correlation value of 0 and 1 , followed by a selection from a pool characterized by a correlation of 2 . Subsequently, this will lead to the transmission of images without an error with more users using any 2-D spatial encoding scheme in general, and the FHSS-2 or FHSS-3 in particular. However, it has to be mentioned that the approach based on FHSS-3 encoding scheme yields a better performance than the FHSS-2 based O-CDMA approach. 


\section{References}

A. A. Shaar and P. A. Davies. A Survey of one-coincidence sequences for frequency-hopped spread-spectrum systems. IEEE Proceedings of Communications, Radar and Signal Processing, 131(7):719-724, 1984. DOI:10.1049/ip-f$1: 19840108$.

A. B. Abche, J-P Toulani, E. Inaty, and E. Karam. Medical Image Transmission Over Multicore Fiber Using Two Stages CDMA Technique. Proceeding of the $5^{\text {th }}$ International Conference on Bioinformatics and Biomedical Engineering, pp. 1-4, May 2011, Wuhan, China. DOI: 10.1109/icbbe.2011.5780447.

C. C. Chang, H. P. Sardesai, and A. M. Weiner. CodeDivision Multiple access Encoding and decoding of Femtosecond Optical Pulses over 2.5-km Fiber Link. IEEE Photonics Technology Letters, 10(1): 171-173, 1998. DOI:10.1109/68.651153.

G-C Yang, and W. C. Kwong, (1998). Image transmission in multicore-fiber code-division multiple access networks. IEEE Transactions on communication, 2(10): 285-287, 1998. DOI: $10.1109 / 4234.725225$.

J. Kohli. Medical Imaging Applications Emerging Broadband Networks. IEEE Communications Magazine, 27(12):8-16, 1989. DOI:10.1109/35.41416.

Po-Rong Chang and C.-C. Chang (1996): Fiber Optic Subcarrier Multiplexed CDMA Local-Area Networks For Subband Image Transmission. IEEE Journal on Selected Areas in Communications, 14(9):1866-1873, 1996. DOI: $10.1109 / 49.545709$.

K. Kamakura and K. Yashiro (2003): An embedded Transmission Scheme Using PPM Signaling with Symmetric Error Correcting Codes for Optical CDMA. Journal of Lightwave technology, 21(7):1601- 1611, 2003. DOI: 10.1109/JLT.2003.814387.

K. Kitayana (1994): Novel Spatial Spread Spectrum based Fiber Optic CDMA Networks For Image Transmission. IEEE Journal On Selected Areas in Commun., 12(4): 762772, 1994. DOI:10.1109/49.286683.

Lisimachos P. Kondi, D. Srinivasan, D. A. Pados, and S. N Batalama. Layered Video Transmission over wireless Multirate DS-CDMA Links. IEEE Transactions on Circuits and Systems for Video technology, 15(12): 16291637, 2005. DOI:10.1109/TCSVT.2005.856922.

L. D. Wronski, R. Hossain, and A. Albicki . Extended Hyperbolic Congruential Frequency Hop Code: Generation and bounds for Cross-and Auto-Ambiguity function. IEEE Transactions on Communication, 44(3): 301- 305, 1996. DOI:10.1109/26.486324.

M. Fuderer .Ringing Artifact Reduction by an Efficient Likelihood Improvement Method. Proceeding of SPIE, Science and Engineering of Medical Imaging, 1137: 8490, 1989. DOI:10.1117/12.961720.

M. Tsiknakis and D, G. Katehakis. Intelligent Image Management in a Distributed PACS and Telemedicine Environment. IEEE Communications Magazine, 34(7): 36- 45, 1996. DOI:10.1109/35.526886.

M. Abtahi and J. A. Salehi. Spread-Space Holographic CDMA Technique: basic Analysis and Applications. IEEE Transactions on wireless Communications, 1(2): 311- 321, 2002. DOI:10.1109/7693.994825.

R. C. Gonzalez and R. Wood. Digital Image Processing. , $3^{\text {rd }}$ edition, Prentice Hall, 2008.

X. Peng, K-B Peng, Z. Lei, F. Chin, and C. C. Ko. Two Layer Spreading CDMA: An Improved Method for Broadband Uplink Transmission. IEEE Transactions on Vehicular Technology, 57(6): 3563-3577, 2008 DOI:10.1109/TVT.2008.919605.

X. Yang, and B. Vucetic. A Frequency Domain Multi-User Detector for TD-CDMA Systems. IEEE Transactions on Communications, 59(9): 2424-2443, 2011. DOI: 10.1109/TCOMM.2011.062111.090546. 